\title{
The Third Dogma Revisited
}

\author{
Petri Ylikoski \\ University of Helsinki \\ petri.ylikoski@helsinki.fi
}

\begin{abstract}
This paper argues that there are some serious problems with the Wesley Salmon's arguments against the so-called third dogma of empiricism. In the first place, his formulation of the dogma is ambiguous: it can be read in at least four different ways. Secondly, his arguments against the dogma are only able to refute the strongest version of it. The second part of the paper considers the idea that covering laws have a constitutive role in singular causal explanation and argues that this idea is not plausible. Consequently, the only defensible form of the dogma is the one that claims that all explanations can be reconstructed as deductive arguments.
\end{abstract}

In this paper I will discuss Wesley Salmon's critique of the third dogma of empiricism. According to this dogma, all scientific explanations are arguments. In the first part I argue that Salmon's thesis is ambiguous, and that his arguments suffice to give up stronger versions of the dogma, but not weaker ones. I suggest a deductivist position according to which explanations can be reconstructed as deductive arguments, but which denies that the deductive relationship has a constitutive role in explanation, is acceptable. The second part of this paper will discuss the role of covering laws in singular causal explanation and argue that the deductivist position should be distinguished from the covering-law theory, since it is not committed to the idea that an explanation always needs a covering law among its premises. After going through several arguments to the effect that a full explanation includes a covering law, I conclude that no convincing arguments have been presented to support the covering-law requirement. To the contrary, I argue, there are several reasons why we should think that singular causal explanations do not include an essential reference to a covering law. However, while I deny that laws have a constitutive role in singular causal explanation, I argue that laws, truisms and other generalizations can have an important heuristic role in the construction of explanations. 


\section{The Third Dogma}

One motivation for the idea that a complete explanation includes or refers to covering laws is a thesis that Wesley Salmon calls 'the third dogma of empiricism'. This 'dogma' says that all scientific explanations are arguments (Salmon 1998, p. 95). The dogma regards scientific explanation as an inference from the statement of the explanans to the statement of the explanandum. The explanation has a deductive structure in which the explanans logically entails the explanandum.

\subsection{Four Versions of the Dogma}

Salmon's definition of the third dogma looks simple, but when one considers his discussion as a whole, the issue becomes more complicated. The dogma can be given various interpretations.

Consider the following theses about complete explanation:

[1] All explanations can be reconstructed as deductive arguments.

[2] Deduction has a constitutive role in explanation.

[3] An explanation involves a deduction from a covering law.

[4] An explanation is nothing but a deduction from one or more covering laws (and the statement of the initial conditions).

Salmon's official definition can be interpreted as claiming [1] or [2], but his arguments against the dogma are directed mainly against [4]. This leaves open the exact content of the dogma. To get started, let us first consider the three arguments Salmon himself presents against the dogma, and let us see where they lead.

Salmon's first argument is based on the observation that the addition of irrelevant information is harmless to an argument, but fatal to an explanation. In deductive logic irrelevant premises do not undermine the validity of the argument. Of course, there is no point in adding such premises, but importantly, they do not affect the validity of the argument. With explanation things are different. It seems that in explanation only relevant information should be included. If one adds to the explanation of the dissolving of a piece of sugar in water the information that the water in question is holy water, one ruins or worsens the explanation. (Salmon 1998, pp. 96-97.)

This argument is less than fatal for a deductivist position. It does not show that explanations are not arguments; it only suggests that explanation involves something more than a simple deductive structure. Salmon's argument works against the position that regards deductive structure as a sufficient condition for explanation and against Hempel's position in particular. But if the supporter of the dogma has some extra conditions for explanation in his analysis, the argument does not work. In such circumstances one can claim that the added irrelevant 
premises are not a part of the explanation. And as they are not parts of the explanation, they cannot make it worse or ruin it.

There is something right in Salmon's observation, but it is not related to explanation per se. Rather, it is related to communication in general. In the delivery of explanatory information, as in all communication of information, the addition of irrelevant information does damage to the message. The recipient of the explanation may not be able to distinguish the information that is intended to be explanatory from the background noise. This general observation has some important consequences for the pragmatics of explanation, but it does not concern the analysis of what makes an explanation explanatory.

Salmon's second argument is based on the idea that explanation requires temporal asymmetry, whereas arguments are not subject to similar constraints. Salmon points out that in common counterexamples to the D-N model, such as in the explanation of the length of the flagpole by the length of its shadow, the requirements of the D-N model do not respect the intuition concerning the temporal asymmetry of the causal explanation. (Salmon 1998, pp. 101-104.)

In principle, this argument works against Hempel's version of the D-N model. However, although it shows that deductive structure is not sufficient for the explanation, it does not show that it is unnecessary. If the supporter of the D-N model adds to her model a requirement concerning causality, as a number of authors have done, she escapes Salmon's critique. Clearly, this argument is only compelling against thesis [4].

\subsection{The Leibniz Principle}

Salmon's third argument concerns the possibility of explaining random events. When explaining outcomes of indeterministic processes, the explanandum is not logically entailed by the explanans. ${ }^{1}$ According to Salmon, the deductive idea connects explanation too closely to the validity of the doctrine of determinism. Developments in physics have shown that the possibility of indeterminism should be taken seriously. In this situation a commitment to the idea of determinism is a clear disadvantage for a theory of explanation. It is more preferable that a theory of explanation remains neutral on this issue and is compatible with the possibility of indeterministic causation. As the scientific evidence seems to strongly support the idea that there are truly indeterministic processes in the world, the deductive ideal of explanation seems to be in trouble. (Salmon 1998, pp. 97-101.)

Again, this is not a knockdown argument against the deductive ideal. A deductivist can reply to Salmon in various ways. One way is to show that a commitment to deductivism does not

\footnotetext{
${ }^{1}$ Salmon also discusses Hempel's inductive-statistical model of explanation. I will not consider it here since I take the very idea of valid inductive argument to be absurd. By accepting inductive-statistical explanations one gives up the deductive ideal of explanation.
} 
automatically imply a commitment to the acceptance of determinism. Salmon argues that a deductivist is committed to the following principle:

If $a$ explains $f$ in one case, and $c$ and $f$ are incompatible, then $a$ cannot explain $c$ in another (similar) case.

Salmon calls this the Leibniz principle (after Stegmüller). According to Salmon, this principle does not hold in the indeterministic cases that are familiar from modern physics (Salmon 1998, pp. 154-158, 326-329). Since giving up the whole idea of explanation in the indeterministic contexts is too high a price to pay, Salmon suggests that we give up the Leibniz principle.

In the following I will argue that there is at least one way to stick to the Leibniz principle and still make sense of explanation in indeterministic contexts. In this approach the explanandum is regarded as having a contrastive structure. An explanation does not explain a plain fact $f$, but a contrastive fact $f$ rather than $c$, where the $c$ is an incompatible alternative to the actual fact $f$ (Ylikoski, 2001). Since the above formulation of the Leibniz principle is in a noncontrastive form, let us reformulate it as follows:

If $a$ explains $f[c]$ in one case, then $a$ cannot explain $c[f]$ in another (similar) case.

Here the notation $f[c]$ should be read as ' $f$ rather than $c$ '.

To understand Salmon's reasoning, let us consider an example. Suppose we have a random device, for example a coin-tossing device, which operates in a truly indeterministic manner. Suppose that the chance of heads will be .65 , and the chance of tails will be .35 . We use the device to throw a coin and get heads $(f)$ instead of tails $(c)$.

Salmon holds that we cannot explain why the result of the throw was heads rather than tails. However, he claims that we can explain why the result was heads. So, we cannot explain why $f[c]$, but we can explain the plain explanandum $f$. (For a similar account see Lewis 1986, pp. 230-231.) This observation convinces Salmon that the Leibniz principle is wrong in indeterministic contexts and that not all explananda are contrastive.

According to Salmon, both the Leibniz principle and the idea that all explanation is contrastive spring from an attachment to a deterministic worldview. If we give up this worldview, on the authority of modern physics, we should also give up these two ideas. (Salmon 1998, pp. 327-328.) I do not share Salmon's assessment of the situation. Remember that we are now speaking about explanation, not causation. It is quite uncontroversial that modern physics describes the world as including irreducibly indeterministic processes. However, I claim that the implications of this fact for the theory of explanation are not that obvious. Salmon needs to show that when we give up the deterministic picture of the world, we also need to give up these two fundamental ideas about explanation. 
Salmon argues for his position by trying to show that we can do without the Leibniz principle. According to him, the main reason for keeping the Leibniz principle sacrosanct is that it rules out some pseudo-explanations. He gives three examples of pseudo-explanations: 1) the explanation of the power of opium to produce sleep by its dormitive virtue; 2) the explanation of all happenings by the will of God; and 3) the explanation of behavior by a psychoanalytic theory that is compatible with all possible behavior. Salmon argues that we don't need the Leibniz principle to show that these explanations are not scientific explanations. According to him, the dormitive virtue theory is too ad hoc to be acceptable, the will of God is a scientifically unacceptable explanation because it refers to a supernatural agency, and psychoanalytic theories which are compatible with all possible behavior cannot be scientifically confirmed. According to him, it suffices that we require that scientific explanations appeal to bona fide scientific laws and theories. (Salmon 1998, pp. 327.) This suggests that the Leibniz principle is only an ad hoc device to rule out pseudo-explanation, not a deep principle of explanation at all. Let us see how his argument fares, before considering whether the Leibniz principle has also some other uses.

It is true that the Leibniz principle is not required to rule out the dormitive virtue theory. However, Salmon's diagnosis of the situation is not accurate either. The explanation is not a pseudo-explanation because it is ad hoc. It is a pseudo-explanation because it is nonexplanatory, and it is non-explanatory because it is uninformative. In contrast, some ad hoc explanations are both informative and explanatory. The trouble with the dormitive virtue explanation is that it simply describes the cause as the kind of cause that causes the effect we are interested in. This explanation does not require any empirical research, and it cannot fail. The explanans is basically deduced from the explanandum, and we do not want explanation to be that easy. ${ }^{2}$

What about God's will as an explanatory factor? Here I think Salmon's diagnosis is ad hoc. It is not enough to claim that the reference to a supernatural agency is not acceptable in science: there should also be some kind of a justification for this claim. We would like to have some principled reason why the God's will is not an acceptable explanatory resource in science. Salmon owes us an acceptable diagnosis of the case that does not presuppose the Leibniz principle. Before that, I think we should stick with the standard justification, which is the following: 'It's God's will' is too easy an explanation. It can explain everything equally well, and it is compatible with everything. Consequently it does not tell us why $f$ happened instead of $c$. Because of this, its explanatory power is only apparent. The scientific theories do not claim to explain everything, but they at least sometimes provide us reasons why $f$ had to occur

\footnotetext{
2 There are some contexts where a reference to dormitive power can be informative and explanatory. If we do not know that opium has such a power and we want know why a person, who has had a dose of opium, fell asleep, the reference to opium's dormitive power is appropriate. However, a reference to a dormitive virtue as an answer to question 'why does opium cause sleep?' is completely unexplanatory.
} 
instead of $c$. We are here basically resting on the Leibniz principle. So, when we appeal to the principle Salmon wants to dispense with, we can make sense of the situation.

A similar solution is applicable to psychoanalytic theories that are compatible with all possible behaviors. (I don't know if there are such psychoanalytic theories, but let us suppose that there are.) If a theory really cannot explain any $f[c]$, it cannot explain anything. The point of scientific theories is to track dependencies in the world, and if a theory does not find any, its explanatory power is zero. We do not need considerations concerning the confirmability of the theory to establish this, as Salmon thinks. And if we do so, we again confront the charge that the reply is ad hoc in nature. We want an account that shows why the theories that are too easy to confirm are not good explanations. And for this purpose we need more fundamental principles, like the Leibniz principle.

Of course, Salmon might just have been too hasty in his analysis of these examples. However, there is a more general reason why his approach to these pseudo-explanations does not work. He thinks that the criterion for ruling out these pseudo-explanations is their unscientific nature. But if the argument rests on this criterion, one needs a criterion of demarcation between science and non-science. Salmon has not provided us any indication as to how this perennial problem can be solved without the Leibniz principle.

Furthermore, it is unclear why we should raise the issue of demarcation at all. An appeal to virtus dormitiva is a bad explanation outside science as well. We should be analyzing what makes explanations good in general. There are good explanations that are not scientific, and there are bad scientific explanations. It would be inconceivable, at least to me, if scientific and everyday explanations did not share some basic ideas about good and bad explanations. After all, science has its origin in common sense cognition. There should be some kind of continuity. Of course, it now includes much that is apparently incompatible with common sense, but I seriously doubt that this incompatibility also extends to the general principles of explanation.

The above considerations suggest that Salmon is not successful in dispensing with the Leibniz principle. It is needed to explain why some pseudo-explanations are not explanatory. The real justification of the principle is that it reflects our conviction that an explanation should trace objective relations of dependency. And since Salmon's ontic conception of explanation is based on this same conviction, it seems clear that he should also stick to something like the Leibniz principle.

\subsection{Explaining Outcomes of Indeterministic Processes}

Does Salmon have any other arguments for dispensing with contrastive explanations and the Leibniz principle? Surprisingly he does not. His discussion is mainly directed against the high probability theories of explanation. According to these theories, we can explain $f[c]$ only if the probability of $f$ is high. By referring to the arbitrariness of any probability threshold for 
explanation and by bringing in some symmetry considerations, he argues that the supporter of the high probability requirement should also accept explanations for the improbable outcomes. I take these arguments to be convincing. If we accept that we can explain $f[c]$ when $f$ has a high probability, we should also accept that it can be explained when $f$ has only a low probability. However, we need some reasons to accept the antecedent of the counterfactual.

Salmon's justification seems to be that if we don't accept the antecedent, we cannot explain indeterministic events at all, and this would be too heavy a price to pay. Fortunately, we do not have to pay this price. The central intuition behind the contrastive approach to explanation is that the proper understanding of the explanation requires thinking carefully about the explanandum. Salmon is right that we can explain something. But the question is: what about them can we explain? And there are a lot of things to explain. For example, we can try explain:

1) why the probability of heads was .35 rather than some other value; or

2) why the result of the experiment was that the coin landed heads or tails, rather than something else, for example, that it melted, was vaporized, etc.

Question 1) directs us to the theoretically interesting case of explaining the propensity of the coin to land heads with some specific probability. Once we have an answer to this question, the lack of an explanation for the outcome of a single experiment is much less important.

Question 2) shows that in principle there are many other contrasts to be explained. I am not claiming that scientists are usually interested in explananda like these, but I think that their possibility can be used to explain Salmon's intuition that we can explain something more about these cases.

Is there anything else to explain? I do not think so. Contrary to Salmon, the contrastive approach is not a relic from the deterministic world that is only a hindrance to the proper understanding of explanation in the indeterministic contexts. Rather, it seems to be an indispensable tool for clarifying what we are capable of explaining in such contexts. Salmon agrees that we cannot completely explain $f$, but his theory does not allow him to explicate what about $f$ can be explained. The contrastive theory can do this, which gives it a clear advantage. In fact, we could claim that a contrastive theory of explanation is something Salmon badly needs in order to save his causal theory of explanation. As Christopher Hitchcock has argued, the central problem in Salmon's approach does not include any considerations of explanatory relevance. The central argument against the covering-law theory has been that it allows irrelevant information to explain. The same charge can be raised successfully against Salmon's theory (Hitchcock 1995, pp. 309-312). The contrastive theory of explanation might help Salmon here. 
Note how little we have to give up to be able to use this tool. We only have to give up the possibility of explaining singular outcomes of experiments (and actual frequencies that are the results of finite series of experiments), when both the fact and the foil have the possibility of occurring. And usually these are not the scientifically most interesting cases. For example, physicists working with quantum theory are not interested in explaining the results of single experiments or actual frequencies of outcomes in particular series of experiments. These actual frequencies are only evidence for the real objects of explanation, which are the probability distributions for the possible outcomes. (Kitcher 1989, pp. 450-452; Woodward 1989, pp. 367-368.) Scientists are interested in the general phenomenon and its probability distributions, not in the idiosyncrasies of some specific set of data. This emphasizes the fact that we can have a deep theoretical understanding of the phenomenon without being able to predict or explain singular outcomes. The belief that the explanation of singular probabilistic outcomes is somehow scientifically central is an artefact of the philosophical discourse on explanation. The problem of the single case is philosophically challenging, but scientifically it is not a big deal.

In a later article, Salmon seems ready to concede this point. He accepts that he cannot convince his opponents about the importance of singular outcomes to theoretical basic science (Salmon 1998, p. 158). Without giving up his position, he tries to show that there are some cases of single-case explanations in the applied sciences that the above strategy cannot handle. He has basically two examples of such situations.

The first example concerns the outbreak of Legionnaires' disease in 1976. All the (original) victims of the disease had stayed in the same hotel, but not all hotel visitors contracted the disease. Actually, only a small percentage did. Later the bacillus responsible for the disease was found in the cooling towers for the air-conditioning system. Salmon suggests that since quantum fluctuations may lead to large uncertainties in the future trajectories of molecules in the air, we might not be able to provide a strictly deterministic explanation for why particular persons contracted the disease and some other people did not contract it in similarly infested rooms. Consequently, we cannot have a deductive explanation why precisely these people contracted the disease. However, he argues that for the purposes of assigning responsibility and taking preventive steps in the future, we have an adequate explanation of the disease in this limited sample of people in 1976. (Salmon 1998, pp. 158-159.)

The second example concerns eight soldiers (out of a group of 2235) who witnessed the detonation of an atomic bomb at close range during Operation Smoky in 1957, and who subsequently developed leukemia. According to Salmon, the high level of radiation they were exposed to explains this incidence. Leukemia occurs with a non-zero frequency in the population at large, and it is possible, but not very likely, that these incidences of leukemia were due to a chance fluctuation rather than exposure to high levels of radiation. This makes the explanation probabilistic. Salmon argues that from a practical standpoint the fact to be 
explained is the high incidence of leukemia in this particular sample, not its incidence among all people who ever have been or will be exposed to that amount of radiation. Consequently, he argues, this is not a deductive statistical explanation. He also argues that this focus on this particular sample was of importance in the deciding whether the soldiers should receive extra compensation from the federal government. (Salmon 1998, pp. 134, 159.)

Salmon's examples, and his arguments, are not convincing. (See Hållsten, 1999 for a similar assessment.) In the case of Legionnaires' disease, we do not need to explain why these particular hotel guests contracted the disease in order to assign responsibility or to take preventive steps. For the latter, it is sufficient to know that there is a risk of Legionnaires' disease with certain kinds of air conditioning machines. It does not matter which individuals contracted the disease or exactly how many instances there were. The central issue is to eliminate the possibility of an outbreak of the disease. Similarly, when attributing responsibility, the significant issue is whether someone is responsible for putting the hotel visitors at risk, not the exact identity or the number of people contracting the disease. We don't have to explain why one particular guest contracted the disease rather than another. All we need to know is that it came from the same source. Consequently, we do not have to explain single cases, we only need to recognize that there is a number of incidences. We can know the cause of their disease without being able to explain why these particular persons contracted it.

Similar points apply to Salmon's leukemia example. The basis of the government's responsibility is in having put the soldiers at a high risk, not causing particular soldiers to have leukemia (Kitcher 1989, p. 459). We do not have to explain the individual cases, we need note only their higher risk of getting this particular form of leukemia. And if a court decides that the risk was high enough, it can decide that the government is responsible, and that it must compensate all soldiers who were put at risk and who developed later leukemia. In this case we cannot know the cause of leukemia in individual cases (as we knew with Legionnaires' disease), but we know what increased their risk of having leukemia. This is enough for attributing legal and moral responsibility.

The above line of reasoning shows that there is no essential connection, or even tension, between the deductive ideal of explanation and the issue of indeterministic causation. One can easily accept both. This makes Salmon's third argument a non-starter. In line with my above analysis, one can accept that some explanations in indeterministic contexts are doomed to be partial or that we cannot explain singular outcomes of indeterministic processes. As my analysis shows, this does not apply to all explananda, so there is much space for Salmon's intuition that we can explain something in the indeterministic contexts. My discussion also shows that the position that is in more trouble is Salmon's own account. After giving up the deductive idea, he really has no resources to explain what it is about the random event that a given explanation explains. The requirement that the statement of the explanandum should be 
logically entailed by the explanans gives a neat way to show the explanatory scope of the explanans. The aspects of the explanandum event that are accessible by deductive reasoning are inside the scope of explanation, and the rest is beyond its reach.

Summing up the arguments so far, Salmon has not been successful in providing good reasons for giving up the third dogma. He has only given us some reasons to give up, or improve, Hempel's theory of explanation. Do we have some other arguments against the dogma? What about van Fraassen's (1980, p. 134) thesis that explanations are not arguments, but answers to explanation-seeking questions? This suggestion is another non-starter. One can accept both ideas and take them to be complementary ideas. An explanation can be an argument and still an answer to an explanation-seeking question, as Hempel saw. Similarly, I think that Achinstein's (1983, pp. 81-83) worries that the D-N account cannot handle the role of emphasis in explanatory statements can be shown to be less than fatal to the deductive ideal of explanation.

But are there any positive motivations for remaining attached to the dogma? After all, if there are no positive reasons for holding the view, even very weak arguments can be enough for giving it up. In my view there is one positive argument for holding on to the dogma. The deductive ideal provides a useful test for the explanatoriness of an answer to an explanationseeking question. One should be able to deduce the statement of the explanandum from premises that include both the statement of the explanans and the background assumptions of the explanation-seeking question. If this is not achieved, the explanation remains partial, no matter what its other merits are. On the other hand, if this test is passed, it is possible that we have an adequate explanation in our hands.

The above considerations show that if the deductive ideal is understood in the sense of thesis [1] (or [2]), it cannot be readily dismissed. Notice also that nothing in the above discussion shows that the acceptance of the deductive ideal commits one to the idea that a singular causal explanation necessarily includes a reference to a covering law or laws. These two ideas should be kept distinct. To see why this is important, let us consider an argument presented by DavidHillel Ruben. The argument shows that the only way to save the motivation for the D-N model is to introduce considerations that destroy the anticipated fruits of this approach.

\subsection{Ruben's Argument}

Ruben starts by the following observation: in order to cope with the standard counterexamples based on irrelevant information, causal preemption and considerations of explanatory asymmetry, the covering law account must be strengthened by including considerations of causality (Ruben 1990, pp. 183-194). But is this enough, and how should it be done? Ruben considers Timothy McCarthy's (1993) argument to the effect that it is not enough to add to Hempel's standard conditions the requirement that there be a premise which mentions the 
actual cause of the event to be explained. McCarthy argues that it is always possible to construct derivations that meet these extended requirements, but which are still not explanatory. To follow Ruben's discussion, let ' $D(e)$ ' be a sentence describing the explanandum $e, ' C(e)$ ' a sentence describing the actual explanatory cause $(c)$ of $e, o$ any object such that $A(o)$, and let ' $(x)(A x->B x)$ ' be any (causal or non-causal) law utterly irrelevant to the occurrence of $e$. Let us now consider the following derivation:

(1) $(x)(A x->B x)$

(2) $C(e) \& A(o)$

(3) $\neg B(o) \vee \neg C(e) \vee D(e)$

(C) $D(e)$

This derivation meets all of Hempel's conditions and also the added requirement that the explanation should mention the cause of the explanandum. Furthermore, as ' $C(e)$ ' has an essential role in the derivation, the example is not arbitrary in this sense. But, as everybody would agree, this argument does not explain $D(e)$. The derivation mentions cause $c$, but it gets us from the cause to the explanandum via a law irrelevant to $c$ 's causing $e$, rather than by asserting that $c$ is the cause of $e$ (McCarthy 1993, p. 130-131). The problem with the example, and others like it, is that the deductive relation between $c$ and $e$ is causally and explanatorily irrelevant to the occurrence of $e$. There is something to the notion of explanatory relevance that even the causally strengthened deductive model cannot capture. (McCarthy, 1993; Ruben 1990, pp. 194-196, 249-251.)

Now, according to Ruben, there is a very simple way to bring the cause and the explanandum together in the right and relevant way. We only have to require that the derivation include as a premise a singular statement that asserts with respect to cause $c$ that it is the cause of $e$. We capture the explanatory dependence by an explicit statement of the causal dependence: ' $c$ caused $e$ '. The premise does not need to use the word 'cause'; it could as well assert that $e$ occurs because $c$ occurs, or that $c$ is the reason for the occurrence of $e$. (Ruben 1990, p. 196, see also Achinstein 1983, chapter 5.)

This suggestion has an important consequence. Hempel's requirement that there be a lawlike generalization in the premises that is essential for the derivation becomes unnecessary. Following Ruben's suggestion we would have a premise that says that the cause of the explanandum event is such-and-such. This premise would entail the statement of the explanandum without any help from the other premises. If the only motivation for the inclusion of law in the explanation is the deductive ideal, Ruben's suggestion makes it redundant. An explanation of a singular fact does not require a generalization as a premise; one can deduce ' $e$ ' from the ' $c$ caused $e$ '. 
For Ruben, this observation has also another interesting consequence. The derivation of ' $e$ ' from ' $c$ caused $e$ ' is trivial in our example, and all the other parts of the derivation are redundant. In the light of this, it is much simpler to think the explanation as consisting of a singular sentence, ' $c$ is the cause of $e$ ' or ' $e$ because of $c$ '. The whole idea of explanations as arguments becomes unmotivated. Ruben concludes that explanations are not arguments, but simply sentences (Ruben 1990, p. 197).

When considering first the destiny of the D-N model, it seems that the required cure kills the patient. The only way to save the covering-law idea leads to the considerations that are its demise. After all, the original point of the approach was to make sense of sentences like ' $e$ because of $c$ '. If the analysans includes the statement of analysandum, the analysis has gone in circles, and the appropriate question is not whether we have advanced, but whether we have been lucky enough to avoid more confusion.

What are the consequences of Ruben's first argument for the supporter of the deductive ideal who does not require the presence of covering laws in the argument? The situation is less clear. Ruben's first argument clearly supports the idea that the deductivist position is distinct from the D-N account. The acceptance of the deductive ideal does not automatically lead to the acceptance of the D-N account of explanation. There is no compulsory move from thesis [1] to thesis [3].

What a deductivist can say about Ruben's second observation? First, it helps us to make difference between the positions subscribing to theses [1] and [2]. They are not necessarily the same thing. Ruben's sentence explanations can be reconstructed as deductive arguments, but deduction is not constitutive of them being explanations. Their explanatory relevance derives from some other source. One can be even more liberal than Ruben. One can allow that we can explain also by pictures, diagrams, gestures and physical models, and still hold that these explanations can, in principle, be reconstructed as deductive arguments.

Second, Ruben's argument does not show that thesis [1] is false. One can accept that in his example deduction is a trivial exercise. This might be true also of more realistic, or less abstract, examples. However, this only applies when we actually have an explanation. If our explanatory information is not really explanatory, the deduction does not go through. This supports the idea that the deductive ideal can still serve as a test for explanatory relevance.

\section{Do Covering Laws Have a Constitutive Role in Explanation?}

The above discussion shows that there are good reasons to think that the deductive ideal does not directly support the idea that singular causal explanation essentially involves reference to a covering-law or generalization. Deductivism and commitment to covering laws in explanation are separate issues. The acceptance of the former does not commit one to the 
latter. But the requirement of covering laws has also been defended on other grounds than the deductive ideal. Now it is time to turn to these arguments.

\subsection{The Arguments for Laws}

We can distinguish three different arguments for the necessity of laws in singular causal explanation. Let us go briefly through these arguments before considering the arguments against this requirement.

The first argument is epistemological. It says that unless we suppose that there are certain uniformities in the nature, we lack justification for our singular causal claims (Hempel 1965, p. 360). The idea is that without the knowledge of the appropriate laws, we would have no grounds for distinguishing between true and false causal claims. Our explanations would lack justification.

Michael Scriven has shown that this argument is not viable. As Scriven insists, we should distinguish between explanatory claims and our grounds for accepting these claims (Scriven 1959, pp. 446-450; 1962, pp. 196-200). It is clear that we can use laws and other generalizations to justify our causal claims. However, this does not imply that we should include these justifications in our explanations. Hempel does not require that we include justification for non-causal claims, so in the name of consistency, why should we include justification for causal claims in the explanation? This asymmetry would require some justification, and no such thing is provided. To the contrary, there are good reasons not to require that the justifications be included in the explanations. First, their inclusion would call for an infinite regress: if we include justifications for the explanatory facts, should we also include justifications for the justifications? There is no natural boundary to stop the regress if it gets started. Secondly, in explanation, we are not looking for reasons for our belief in the explanandum: we presume that it is the case, and we are asking why it is so. Similarly, explanation presumes that the facts it cites are true, it does not look for justification for our belief in them. Our explanation explains if it is true; the grounds for believing it are a separate question.

The second argument for the necessity of laws in causal explanation is semantic. It states that causal explanatory claims tacitly imply that there are appropriate laws that cover them (Hempel 1965, p. 349). Again, I think that there are good reasons for not going along with this argument. It seems to presuppose that some kind of regularity account of causation is correct. But this presupposition is clearly unjustified: it is not the case that a singularist account of causation is wrong on purely semantic grounds. To the contrary, it seems that most philosophers of causation nowadays regard regularity accounts of causation as highly problematic and suspect. It would be unfortunate to bind the account of explanation to such a weakly supported metaphysical position. And if this is to be done, it should not be done on 
purely semantic grounds. Secondly, even if a causal explanation would imply that there is an appropriate covering law, this would not necessarily imply that this covering law has $a$ constitutive role in the explanation. The supporter of Hempel's position would have to present some argument to the effect that covering laws are not just epiphenomenal on explanatory causes. The only way to do this would be to claim that the regularity constitutes the causality, which would bring us back to the problematic empiricist account of causation. Clearly, the semantic argument is as unconvincing as the epistemological one.

The third, and the final, positive argument is based on the ideal form of scientific knowledge. This is the primary motivation for Peter Railton's DNP model of explanation. According to Railton, "the fundamental insight of the covering-law approach to scientific explanation is that science seeks theoretical understanding, guided by a nomothetical ideal" (Railton 1980, p. 117). The basic goal of science in this account is the subsumption of particular facts and regularities to the 'nomic nexus'. This is achieved by fitting the world's phenomena into a fully general and comprehensive theory (Railton 1980, pp. 117-119.) In Railton's vision, the ideal for which science strives is the 'ideal explanatory text', which would be able to explain every aspect of the phenomenon under consideration and would have a deductive-nomological structure. He notes, that “... plainly there is no question of ever setting such an ideal text on paper" (Railton 1981, p. 247), but he wants to underline the regulative role of such ideal. The aim of science is to develop a capacity to provide material for such ideal explanatory texts.

I do not want discuss the merits of Railton's ideal as a description of the goal of scientific knowledge. Clearly, he describes a picture of science that is accepted by many philosophers of science. Supposedly the ideal has also motivated quite a few covering-law theorists. However, this picture does not in any way show that laws have a constitutive role in actual explanations. One could agree with everything Railton says and hold that the fundamental aim of science is to create a general theoretical conception that reveals the nomological structure of the world, but still maintain that laws do not have a constitutive role in the singular causal explanation. Actually, this seems to be even Railton's own position, since he holds that we can perfectly well explain without recourse to covering laws (Railton 1981, p. 249).

Clearly, the question concerning the ideal form of scientific knowledge is a separate issue from the question 'what makes a given explanation explanatory?' The idea of the ideal explanatory text does not give us any hint about the principles that govern the choice of explanatory information. It requires an addition from some other theory of explanation to be able to handle this central problem. On the other hand, the contrastive approach to explanation can be used to characterize the concept of the ideal explanatory text (Ylikoski 2001, p. 25). The elements of the ideal explanatory text for event $e$ are explanatory because they are parts of adequate answers to some contrastive questions about $e$. This asymmetry between the two accounts of explanation suggests that the contrastive approach is conceptually more fundamental. 
Careful consideration of these arguments shows that the positive grounds for the belief that laws have a constitutive role in singular causal explanation have always been quite shaky. The position should have somewhat stronger grounds for it to be taken seriously. Contrary to a common presumption, the burden of proof is with the supporter of the covering-law account, not with its opponent. Furthermore, there are weighty arguments against the idea. Let us next consider these arguments briefly.

\subsection{The Arguments Against Laws}

First, consider the fact that our everyday explanations do not mention laws. This also goes for most explanations in the sciences. Furthermore, laws are not even tacitly implied by these explanations, since often people do not know the appropriate laws. Either the laws are totally unknown, or we do not know them in the form that would allow us to make appropriate derivations. These facts are something that the supporters of the D-N model grant to their critics, but I think that they have not considered all the implications of this observation.

Consider next James Woodward's plausible desideratum for a theory of explanation:

A theory of singular causal explanation ought to identify the structural features of such explanations which function so as to produce understanding in the ordinary user. Features which are entirely unknown to the ordinary user are not plausible candidates for what produces such understanding. (Woodward 1993, p. 249)

According to this criterion, the features that produce explanatory understanding should be epistemically accessible. (Woodward 1986, p. 269) The problem for the covering-law account is that it requires that the explanandum be deduced from general laws and a description of the initial conditions. Now, as both the law and the deductive argument are ordinarily unknown, the question is how does the ordinary explainer identify the explanatory factors and separate them from the non-explanatory factors? Does she have some sort of intuition that recognizes the existence of these unknown factors, or is she just guessing? The first sounds mystical and the second implausible. The supporter of the D-N account owes us 1) an explanation for how we can judge explanatory relevance of causal claims and 2) an account of the relation between the principles behind these judgments and the conditions spelled out by her model. I do not claim that this is impossible. I just wish note that no one has done it. As long as these questions remain without answers, the covering-law account cannot be taken as a serious explication of the ordinary notion of (scientific) explanation. Of course, it is always possible to take the stipulative strategy and treat the covering-law account as a definition of explanation, but what would be the point of that exercise?

There are also some other difficulties. One clear problem is that apparently there are no covering laws for most explananda. For example, Hempel owes us a justification for the 
assumption that there are enough covering laws for all acceptable explanations. Recall that he says that explanation is always under a certain description, but he does not say that we can only explain things that are described by using the vocabulary of the theoretical sciences. His examples of explanation make use of concepts and descriptions that come from our everyday affairs rather than from the theoretical sciences. The problem is that the sciences do not contain laws that cover all possible descriptions that we might wish to use in our explanatory questions. It is controversial whether so-called special sciences have laws, and if they have, what kind of laws they are, but most people agree that there are no laws for every description. We do not have laws about shattering windows, lighting matches, or failing carburetors, as described. Even when we do have some 'laws', they are not of the right kind. These laws use descriptions that are alien to our explananda, and consequently, they are not capable of functioning in logical derivations as Hempel wishes they would.

A scientific explanation that intends to make use of laws, or that aims to give an informative theoretical account of the explanandum, must redescribe, or reconstruct, the explanandum in its special theoretical vocabulary (Woodward 1979, p. 62). If we want derivations from scientific laws, we have to describe the explanandum appropriately. And this reconstruction will, at least sometimes, change the explanandum, so that we are not necessarily explaining the same thing anymore. Hempel faces a dilemma here: either he has to restrict the scope of explanations to the explananda that are using the vocabulary of our theoretical sciences, or he has to provide us with an account of these other covering 'laws'. This account would have to justify the scientific status of these 'laws', to show that they are available, and finally to show that there is a point in calling these generalizations laws (Scriven 1959). All this work still remains to be done. There is an obvious reason for this: there is still no consensus about the whole notion of law of nature. As long as this issue remains open, the most substantial part of the D-N account remains open.

To add one more item to the list of things that covering-law theorist should explain, consider our everyday intuition that causation is a local process. The Titanic sank because it collided with an iceberg. The covering-law theorist would like us to explain this unfortunate incident by showing that there is a law to the effect that all the boats similar to Titanic sink when they collide with icebergs (that are similar to the iceberg that Titanic collided with) in an appropriate way. My concern now is not with the form of this law, but with its function in the explanation. The question to ask is how the knowledge that all similar boats sink under these circumstances helps us to understand why the Titanic sank $?^{3}$ Our original question concerns

\footnotetext{
${ }^{3}$ Note the following difference between a covering-law theorist and a supporter of the contrastive approach. The covering-law theorist is interested in other sinking ships because they provide confirmation for his law claim. The supporter of contrastive approach is also interested in other ships, but she does not want information of the exactly similar ships in the precisely same circumstances as the covering-law theorist does. She would like to know about slightly different ships in the same circumstances or about similar ships in different circumstances in order to find interesting differences to be explained.
} 
one particular local causal process, and the explanation of the covering-law theorist refers to other similar causal processes that have no connection to the process we are interested in. How can noting that there are (or can be) similar incidents make us understand this particular incident? I just do not understand. If one accepts an empiricist account of laws, then the generalization is simply a summary of particulars. What emergent explanatory resources does the generalization have that its instances lack (for an empiricist like Hempel)? (See also Ruben 1990, p. 204.) As I see the situation, we do not need laws to explain why the Titanic sank. We only need to know the particular causes at work in this particular case.

Of course, we can further ask why, or how, ships like the Titanic sink when they collide with an iceberg. Indeed, this seems to be the kind of question that pure basic science tries to answer. My claim that we do not need laws to explain singular events does not imply that laws are completely uninteresting or that they cannot be used to explain anything. Apart from explaining singular occurrences, science also aims to uncover regularities in nature and to explain them. Notice that when we explain why ships like the Titanic sink when they collide with an iceberg, the covering-law theorist's generalization reappears. However, it is important to recognize that its not a part of the explanans: it works as the explanandum. This is quite typical in special sciences: the 'covering laws' are things to be explained, not things providing explanatory understanding (Cummins 2000, pp. 119-122).

These considerations strongly support the position that does not take laws to have a constitutive role in the singular causal explanation. Laws (or generalizations) have a more essential role in other kinds of explanation, but these cases have not been the topic of my discussion. I am not claiming that there are no scientific explanations that are derivations from laws. Evidently there are such things. Neither am I claiming that scientific explanations cannot be represented as arguments. To the contrary, I accept this thesis. For example, there might be some good pedagogical reasons to represent an explanation as an argument and in this way display its tacit presuppositions. It is also true that when one's science is privileged enough to have quantitative theories, a derivation is a natural way of demonstrating the dependence between the explaining factors and the factors to be explained. This is the only way to represent precisely the quantitative relations between the variables. This rationale is missing from the usual examples of covering-law explanation. In the first place, the laws in these explanations are non-quantitative. Second, they are singular explanations, whereas scientific derivations concern law-like generalizations. Third, the laws in Hempel's examples are usually mere generalizations, not statements of functional interdependence. They do not allow the derivation of other explananda that are appropriately different from the actual explanandum. (Woodward 1979, pp. 46-49.)

\subsection{An Alternative Role for Laws in Singular Explanation}


In the above discussion, laws play a minimal role in singular causal explanation. However, there are roles for laws in explanation other than being parts of it. First, we have already mentioned their role in justifying singular causal claims. But there is also another important role. It concerns the discovery of explanatory causes. The central function of theories in the sciences is not to provide generalizations or 'laws' that cover individual types of separate events. Rather, it is to serve as general schemes for constructing explanations. Theories are not just collections of covering laws, but accounts of systematic dependencies between the variables. As far as singular causal explanations are concerned, the utility of theories is that they allow an easy and convenient way to construct explanations. With a good theory, all one needs to do is to check the initial conditions and parameters, and then ensure that the provisos of the theory are satisfied; and the theory will then lead one to the explanatory causes. The generality of theories does not make them explanatory, rather it makes them very useful in constructing explanations.

This observation suggests a positive role for various truisms, rules of thumb, tendency statements and other generalizations that are found in the social sciences. Their central role is not to work as premises of an explanatory argument, but rather to work as heuristic rules for the search of explanations. They tell us where to look for the explanatory factors. These generalizations apply only to limited domains and can include numerous exceptions, which makes them less than laws, but they can still be useful. They tell us what kinds of causes are worth looking for. And if we can spell out the exceptions and the intervening factors, we know what kind of factors to look for when the truism does not seem to apply. Consequently, the extreme ceteris paribus nature of these generalizations is not a problem. As their role is heuristic, and not explanatory, the fact that they are not really laws does not matter.

\section{Conclusion}

In this paper I have argued that there are some serious problems with Salmon's arguments against what he calls the third dogma of empiricism. In the first place, his formulation of the dogma is ambiguous: it can be read in at least four different ways. Secondly, his arguments against the dogma are only able to refute the strongest version of it. However, this result should not be considered as a victory for the covering law account of explanation. As my arguments in the second part show, the idea that covering laws have a constitutive role in singular causal explanation is not plausible. The only defensible form of the dogma is the one that claims that all explanations can be reconstructed as deductive arguments.

\section{References}

Achinstein, Peter: 1983, The Nature of Explanation. Oxford: Oxford University Press. 
Cummins, Robert: 2000, "How Does It Work?" versus "What Are the Laws?": Two Conceptions of Psychological Explanation. In Keil \& Wilson (eds.): Explanation and Cognition. Cambridge: MIT Press, pp. 117-144.

Hempel, Carl: 1965, Aspects of Scientific Explanation. New York: The Free Press.

Hitchcock, Christopher C.: 1995, Discussion: Salmon on Explanatory Relevance. In Philosophy of Science 62: 304-320.

Hållsten, Henrik: 1999, Deductive Chauvinism. In Synthese 120: 49-59.

Kitcher, Philip: 1989, Explanatory Unification and the Causal Structure of the World. In Kitcher \& Salmon (eds.): Scientific Explanation. Minnesota Studies in the Philosophy of Science vol XIII. Minneapolis: University of Minnesota Press, pp. 410-505.

Lewis, David: 1986, Philosophical Papers vol II. Oxford: Oxford University Press.

McCarthy, Timothy: 1993, On an Aristotelian Model of Scientific Explanation. In Ruben (ed.): Explanation. Oxford: Oxford University Press, pp. 128-135.

Railton, Peter: 1978, A Deductive-Nomological Model of Probabilistic Explanation. In Philosophy of Science 45: 206-226.

Railton, Peter: 1980, Explaining Explanation: A Realist Account of Scientific Explanation. Ph.D. thesis. Princeton University.

Railton, Peter: 1981, Probability, Explanation, and Information. In Synthese 48: 233-256.

Ruben, David-Hillel: 1990, Explaining Explanation. London: Routledge.

Salmon, Wesley: 1998, Causality and Explanation. Oxford: Oxford University Press.

Scriven, Michael: 1959, Truisms as the Grounds for Historical Explanations. In Gardiner (ed.): Theories of History. New York: The Free Press, pp. 443-475.

Scriven, Michael: 1962, Explanations, Predictions, and Laws. In Feigl \& Maxwell (eds.): Scientific Explanation, Space, and Time. Minnesota Studies in the Philosophy of Science vol III. Minneapolis: University of Minnesota Press, pp. 170-230.

van Fraassen, Bas: 1980, Scientific Image. Oxford: Oxford University Press.

Woodward, James: 1979, Scientific Explanation. In The British Journal for the Philosophy of Science 30: 41-67.

Woodward, James: 1986, Are Singular Explanations Implicit Covering-Law Explanations. In Canadian Journal of Philosophy 16: 253-280.

Woodward, James: 1989, The Causal Mechanical Model of Explanation. In Kitcher \& Salmon (eds.): Scientific Explanation. Minnesota Studies in the Philosophy of Science vol XIII. Minneapolis: University of Minnesota Press, pp. 357-383.

Woodward, James: 1993, A Theory of Singular Causal Explanation. In Ruben (ed.): Explanation. Oxford: Oxford University Press, pp. 246-274.

Ylikoski, Petri: 2001, Understanding Interests and Causal Explanation. Ph.D.-thesis, Department of Social and Moral Philosophy. University of Helsinki. Available at http:// ethesis.helsinki.fi/julkaisut/val/kayta/vk/ylikoski/ 